\title{
Nonrelativistic conformal transformations in Lagrangian formalism
}

\author{
K. Andrzejewski* J. Gonera, A. Kijanka-Dec \\ Department of Theoretical Physics and Computer Science, \\ University of Łódź, \\ Pomorska 149/153, 90-236 Łódź, Poland
}

\begin{abstract}
The conformal transformations corresponding to $N$-Galilean conformal symmetries, previously defined as canonical symmetry transformations on phase space, are constructed as point transformations in coordinate space.
\end{abstract}

\section{Introduction}

In the recent paper [1] we have constructed, using the orbit method [2], the general Hamiltonian system on which the centrally extended $N$-Galilean conformal algebra [3] (group) acts transitively as symmetry group (the central extension is possible for $N$ - odd in any space-time and for all $N$ in $(2+1)$ dimensional one [4]-[6]). It appears that any such system consists of "external" variables forming standard phase space and the "internal" ones: spin (related to $S U(2)$ subgroup) and pseudospin (related to $S L(2, \mathbb{R})$ subgroup). The dynamics of external and internal variables are decoupled. Neglecting the internal variables one obtains the free dynamics governed by higherderivative theory [6] (in fact, the necessity of considering higher-derivative theory was revealed in [7, 8]).

*e-mail: k-andrzejewski@uni.lodz.pl 
In Ref. 1] the conformal transformations were introduced as canonical symmetry transformations acting on phase manifold. Here we complete the picture by showing that, within the formalism of higher-derivative theories, the conformal transformations can be defined as point transformations acting on configuration space. Using the results of Ref. [1] we derive the form of point transformations and show that the higher-derivative Lagrangian describing free motion is invariant (up to a total derivative) under the action of these transformations.

The paper is organized as follows. In Section 2 we remind the main results of Ref. [1] and derive the explicit form of canonical symmetry transformations. In Section 3 and 4 the point transformations on configuration space are defined which coincide "on shell" with these introduced in Section 2 and it is shown that they are Noether symmetries of free higher-derivative Lagrangian with integrals of motion corresponding to the ones on Hamiltonian level (in order to do this we construct an integral of motion for an arbitrary higher-order Lagrangian). Section 5 is devoted to concise conclusions. Some technicalities are relegated to the Appendix.

\section{Hamiltonian formalism}

The $N$-Galilean conformal algebra has the following structure. First, we have the direct sum of $s u(2)$ algebra (spanned by $J_{k}$ 's) and $s l(2, \mathbb{R})$ one (spanned by $H, D$ and $K$ )

$$
\begin{aligned}
{\left[J^{a}, J^{b}\right] } & =i \epsilon_{a b c} J^{c}, \\
{[D, H]=i H, \quad[D, K] } & =-i K, \quad[K, H]=2 i D .
\end{aligned}
$$

It is supplemented by $3(N+1)$ (in general case of $d$-dimensional space $d(N+1))$ dimensional abelian algebra which carries $D^{\left(1, \frac{N}{2}\right)}$ representation of $S U(2) \times S L(2, \mathbb{R})$ and is spanned by the generators $C_{i}^{a}, a=1,2,3 ; i=$ $0,1, \ldots, N$. The relevant commutation rules involving $C_{i}^{a}$ read

$$
\begin{aligned}
{\left[J^{a}, C_{j}^{b}\right] } & =i \epsilon_{a b c} C_{j}^{c}, \quad\left[H, C_{j}^{a}\right]=-i j C_{j-1}^{a}, \\
{\left[D, C_{j}^{a}\right] } & =i\left(\frac{N}{2}-j\right) C_{j}^{a}, \quad\left[K, C_{j}^{a}\right]=i(N-j) C_{j+1}^{a} .
\end{aligned}
$$

For $N$ odd (and also $N$ even in $2+1$ dimensions) the algebra defined by eqs. (11) and (2) admits the central extension [5, 6].

$$
\left[C_{j}^{a}, C_{k}^{b}\right]=i \delta_{a b} \delta_{N, j+k}(-1)^{\frac{k-j+1}{2}} j ! k ! M,
$$


with $M$ being additional central generator. The above algebra can be integrated to the group $(S U(2) \times S L(2, \mathbb{R})) \ltimes \mathbb{R}^{3 N+4}$ where $\mathbb{R}^{3 N+4}$ is nilpotent group and the semidirect product is defined by the $D^{\left(1, \frac{N}{2}\right)} \oplus D^{(0,0)}$ representation of $S U(2) \times S L(2, \mathbb{R})$.

The question arises what are the dynamical systems exhibiting the symmetry described by $N$-Galilean conformal group. In the case of centrally extended algebra the answer was given in Ref. [6]. The results obtained there have been generalized in Ref. [1] to the space of arbitrary Hamiltonian system on which our group acts transitively. Below we discuss the properties of the system constructed by Gomis and Kamimura [6]; the general case differs by the existence of additional internal degrees of freedom [1].

The phase space is parametrized by the canonical variables $q_{k}^{a}, p_{k}^{a}, a=$ $1,2,3, k=0,1 \ldots, \frac{N-1}{2}$, obeying

$$
\left\{q_{i}^{a}, p_{k}^{b}\right\}=\delta_{a b} \delta_{i k}
$$

Defining

$$
\begin{aligned}
h(t)= & \frac{1}{2 m}\left(\vec{p}_{\frac{N-1}{2}}\right)^{2}+\sum_{k=1}^{\frac{N-1}{2}} \vec{q}_{k} \vec{p}_{k-1}, \\
d(t)= & \sum_{k=0}^{\frac{N-1}{2}}\left(\frac{N}{2}-k\right) \vec{q}_{k} \vec{p}_{k}, \\
k(t)= & \frac{m}{2}\left(\frac{N+1}{2}\right)^{2}\left(\vec{q}_{\frac{N-1}{2}}\right)^{2}-\sum_{k=0}^{\frac{N-3}{2}}(N-k)(k+1) \vec{q}_{k} \vec{p}_{k+1}, \\
\vec{j}(t)= & \sum_{k=0}^{\frac{N-1}{2}} \vec{q}_{k} \times \vec{p}_{k},
\end{aligned}
$$

one finds the Noether charges corresponding to the generators of Lie algebra 
(11)-(3)

$$
\begin{aligned}
& h=h(t), \\
& d=d(t)-t h(t), \\
& k=k(t)-2 t d(t)+t^{2} h(t), \\
& \vec{j}=\vec{j}(t), \\
& \vec{c}_{j}=\left\{\begin{array}{c}
(-1)^{j-\frac{N-1}{2}} \sum_{k=0}^{j} \frac{j !}{(j-k) !} t^{j-k} \vec{p}_{k}, \quad 0 \leq j \leq \frac{N-1}{2}, \\
(-1)^{j-\frac{N-1}{2}} \sum_{k=0}^{\frac{N-1}{2}} \frac{j !}{(j-k) !} t^{j-k} \vec{p}_{k}+ \\
m \sum_{k=\frac{N+1}{2}}^{j}(-1)^{j-k} \frac{j !}{(j-k) !} t^{j-k} \vec{q}_{N-k}, \quad \frac{N+1}{2} \leq j \leq N .
\end{array}\right.
\end{aligned}
$$

These charges generate the canonical transformations representing the $N$ Galilean conformal group on Hamiltonian level. Computing systematically the infinitesimal action of all generators we find:

- for $\vec{c}_{j}$ :

$$
\begin{aligned}
& \delta \vec{q}_{n}=\left\{\sum_{j} \vec{x}_{j} \vec{c}_{j}, \vec{q}_{n}\right\}=\sum_{k=0}^{N-n}(-1)^{k+n-\frac{N+1}{2}} \frac{(k+n) !}{k !} t^{k} \vec{x}_{k+n}, \\
& \delta \vec{p}_{n}=\left\{\sum_{j} \vec{x}_{j} \vec{c}_{j}, \vec{p}_{n}\right\}=m \sum_{k=0}^{n}(-1)^{k} \frac{(k+N-n) !}{k !} t^{k} \vec{x}_{k+N-n},
\end{aligned}
$$

- for $h$ :

$$
\begin{aligned}
& \delta \vec{q}_{n}=\left\{\tau h, \vec{q}_{n}\right\}=-\tau\left(\frac{1}{m} \delta_{\frac{N-1}{2}, n} \vec{p}_{\frac{N-1}{2}}+\left(1-\delta_{\frac{N-1}{2}, n}\right) \vec{q}_{n+1}\right), \\
& \delta \vec{p}_{n}=\left\{\tau h, \vec{p}_{n}\right\}=\tau\left(1-\delta_{n 0}\right) \vec{p}_{n-1},
\end{aligned}
$$

- for $d$ :

$$
\begin{aligned}
& \delta \vec{q}_{n}=\left\{\lambda d, \vec{q}_{n}\right\}=\lambda\left(-\left(\frac{N}{2}-n\right) \vec{q}_{n}+t\left(\frac{1}{m} \vec{p}_{\frac{N-1}{2}} \delta_{\frac{N-1}{2}, n}+\left(1-\delta_{\frac{N-1}{2}, n}\right) \vec{q}_{n+1}\right),\right. \\
& \delta \vec{p}_{n}=\left\{\lambda d, \vec{p}_{n}\right\}=\lambda\left(\left(\frac{N}{2}-n\right) \vec{p}_{n}-t\left(1-\delta_{n 0}\right) \vec{p}_{n-1}\right)
\end{aligned}
$$


- for $k$ :

$$
\begin{aligned}
& \delta \vec{q}_{n}=\left\{c k, \vec{q}_{n}\right\}=c\left(\left(1-\delta_{n 0}\right) n(N-n+1) \vec{q}_{n-1}+\right. \\
&\left.2 t\left(\frac{N}{2}-n\right) \vec{q}_{n}-t^{2}\left(\frac{1}{m} \delta_{n, \frac{N-1}{2}} \vec{p}_{\frac{N-1}{2}}+\left(1-\delta_{n, \frac{N-1}{2}}\right) \vec{q}_{n+1}\right)\right) \\
& \delta \vec{p}_{n}=\left\{c k, \vec{p}_{n}\right\}=c\left(m \delta_{n \frac{N-1}{2}}\left(\frac{N+1}{2}\right)^{2} \vec{q}_{\frac{N-1}{2}}-\left(1-\delta_{n \frac{N-1}{2}}\right)(N-n)(n+1) \vec{p}_{n+1}-\right. \\
&\left.2 t\left(\frac{N}{2}-n\right) \vec{p}_{n}+t^{2}\left(1-\delta_{n 0}\right) \vec{p}_{n-1}\right) .
\end{aligned}
$$

These equations can be integrated out to yield the global transformations.

\section{Lagrangian formalism}

We want to find the realization of $N$-Galilean conformal group as a group of symmetry transformations on coordinate space (point transformation). Let us note that the Hamiltonian $h$ given by first eq. (5) is the Ostrogradski Hamiltonian [10] corresponding to higher derivative free Lagrangian

$$
L=\frac{m}{2}\left(\frac{d^{\frac{N+1}{2}} \vec{q}}{d t^{\frac{N+1}{2}}}\right)^{2} .
$$

The procedure of passing from Lagrangian to Hamiltonian formalism is slightly involved [10]. First, one enlarges the coordinate space by defining:

$$
\vec{q}_{0}=\vec{q}, \quad \vec{q}_{1}=\dot{\vec{q}}, \ldots, \vec{q}_{\frac{N-1}{2}}=\vec{q}^{\left(\frac{N-1}{2}\right)}
$$

then one writes out the Lagrangian

$$
\tilde{L}=\frac{m}{2}\left(\frac{d \vec{q}_{\frac{N-1}{2}}}{d t}\right)^{2}+\sum_{k=0}^{\frac{N-3}{2}} \vec{\lambda}_{k}\left(\dot{\vec{q}}_{k}-\vec{q}_{k+1}\right)
$$

$\tilde{L}$ is singular so the Dirac method has to be applied. It appears that all resulting constraints are of the second class which allows to eliminate the Lagrange multipliers $\vec{\lambda}_{k}$ and their conjugate momenta. In this way we arrive at the Ostrogradski Hamiltonian. 
To find the action of $N$-Galilean conformal group in coordinate space let us remind that the general canonical transformation describes the point transformation provided the new coordinates are expressible in terms of old ones (with no momenta involved) while the new momenta are the linear functions of old momenta (with coordinate-dependent coefficient); actually they can be more general affine functions if the Lagrangian transforms by a total derivative. However, the above statement is true only provided the time variable remains unchanged. If it changes the momenta can enter the expressions for the variations of coordinate variables provided they appear only in the form of Poisson brackets of Hamiltonian with coordinates. Then the terms containing momenta can be removed from transformation formulae at the expense of admitting the time variation. The resulting modified point transformations coincide "on shell" with the initial canonical ones.

A simple inspection of the formulae given in Sec. 2 shows that the canonical action of $N$-Galilean conformal group has the above mentioned properties. Therefore, one can define the action of this group on coordinate space which "on-shell" coincides with the transformations defined in Sec. 2. It is not difficult to derive the form of this action. First, let us note that the relations (17)-(10) yield immediately the global form of transformations generated by $\vec{c}_{k}$ 's:

$$
\begin{aligned}
t^{\prime} & =t \\
\overrightarrow{q_{n}^{\prime}}\left(t^{\prime}\right) & =\vec{q}_{n}(t)+\sum_{k=0}^{N-n}(-1)^{k+n-\frac{N+1}{2}} \frac{(k+n) !}{k !} t^{k} \vec{x}_{n+k} .
\end{aligned}
$$

The global action of $h$ obviously reads

$$
\begin{aligned}
t^{\prime} & =t+\tau, \\
\overrightarrow{q_{n}^{\prime}}\left(t^{\prime}\right) & =\vec{q}_{n}(t) .
\end{aligned}
$$

To find the action of dilatation we rewrite eq. (9) in the form

$$
\delta \vec{q}_{n}=\lambda\left(n-\frac{N}{2}\right) \vec{q}_{n}+\lambda t \dot{\vec{q}}
$$

which can be easy integrated to

$$
\begin{aligned}
t^{\prime} & =e^{-\lambda} t \\
\overrightarrow{q_{n}^{\prime}}\left(t^{\prime}\right) & =e^{\lambda\left(n-\frac{N}{2}\right)} \vec{q}_{n}(t) .
\end{aligned}
$$


The case of conformal transformation is slightly more involved. First, by extracting the coefficient in front of $h(t)$ in the expression defining $k$ we find

$$
\delta t=c t^{2}
$$

which integrates to

$$
t^{\prime}=\frac{t}{1-c t} \equiv t(c)
$$

Now, the first eq. (10) can be written in the form

$$
\delta \vec{q}_{n}=c\left(n(N-n-1) \vec{q}_{n-1}+2 t\left(\frac{N}{2}-n\right) \vec{q}_{n}-t^{2} \dot{\vec{q}}_{n}\right) .
$$

The last term on the right-hand side is responsible for time variation. One can get rid of this term by replacing the time variable by its "running" value (19). In this way we arrive at the following equations

$$
\frac{d \vec{q}_{n}(t(c), c)}{d c}=n(N-n+1) \vec{q}_{n-1}(t(c), c)+\frac{2 t}{1-c t}\left(\frac{N}{2}-n\right) \vec{q}_{n}(t(c), c) .
$$

It is not difficult to integrate eq. (21). The result reads

$$
\overrightarrow{q_{n}^{\prime}}\left(t^{\prime}\right)=\sum_{k=0}^{n}\left(\begin{array}{l}
n \\
k
\end{array}\right) \frac{(N+k-1) !}{(N-1) !} \frac{c^{k}}{(1-c t)^{N+k}} \vec{q}_{n-k}(t) .
$$

Finally, the action of rotation subgroup is standard.

Let us note that in all cases the following important property holds:

$$
\vec{q}_{n+1}=\dot{\vec{q}}_{n} \quad \text { implies } \quad{\overrightarrow{q^{\prime}}}_{n+1}\left(t^{\prime}\right)=\frac{d \overrightarrow{q_{n}^{\prime}}\left(t^{\prime}\right)}{d t^{\prime}}
$$

It allows us to reduce the action of the group under consideration to that on the variables $t$ and $\vec{q}=\vec{q}_{0}$. One finds

$$
\begin{gathered}
t^{\prime}=t, \quad \overrightarrow{q^{\prime}}\left(t^{\prime}\right)=\vec{q}(t)+\sum_{k=0}^{N}(-1)^{k-\frac{N+1}{2}} t^{k} \vec{x}_{k} \\
t^{\prime}=t+\tau, \quad \overrightarrow{q^{\prime}}\left(t^{\prime}\right)=\vec{q}(t) \\
t^{\prime}=e^{-\lambda} t, \quad \overrightarrow{q^{\prime}}\left(t^{\prime}\right)=e^{-\lambda \frac{N}{2}} \vec{q}(t)
\end{gathered}
$$




$$
t^{\prime}=\frac{t}{1-c t}, \quad \overrightarrow{q^{\prime}}\left(t^{\prime}\right)=\frac{\vec{q}(t)}{(1-c t)^{N}}
$$

as the counterparts of eqs. (14) (15) (17) (12) (22), respectively.

It is shown in Appendix that in all cases $L d t$ (with $L$ given by eq. (15)) is invariant, up to an exact differential, under all the above transformations.

Eqs. (24)-(27) allow us to write out the differential realization of the algebra (11), (2). It reads

$$
\begin{array}{r}
\hat{H}=i \frac{\partial}{\partial t}, \quad \hat{D}=-i\left(\frac{N}{2} \vec{q} \frac{\partial}{\partial \vec{q}}+t \frac{\partial}{\partial t}\right), \\
\hat{K}=i\left(N t \vec{q} \frac{\partial}{\partial q}+t^{2} \frac{\partial}{\partial t}\right), \hat{\vec{C}}_{k}=i(-1)^{k-\frac{N-1}{2}} t^{k} \frac{\partial}{\partial \vec{q}} .
\end{array}
$$

Let us also note that $\hat{\vec{C}}_{k}$ commutate with each other. This is due to the fact that $M$, being central element, act trivially in coadjoint representation.

In order to complete the picture we will find all integrals of motion corresponding to the transformations (24)-(27) and compare them with the ones defined on the Hamiltonian level (6) . First, let us note that for infinitesimal transformations

$$
\overrightarrow{q^{\prime}}=\vec{q}+\epsilon \vec{\chi}(q, t), \quad t^{\prime}=t+\epsilon g(t)
$$

and an arbitrary higher-order Lagrangian $L=L\left(\vec{q}, \dot{\vec{q}}, \ldots, \vec{q}^{(R)}\right), R>1$, the symmetry condition

$$
L\left(\overrightarrow{q^{\prime}}\left(t^{\prime}\right), \frac{d \overrightarrow{q^{\prime}}}{d t^{\prime}}, \ldots, \frac{d^{R} \overrightarrow{q^{\prime}}}{d t^{\prime}}\right) \frac{d t^{\prime}}{d t}=L\left(\vec{q}(t), \frac{d \vec{q}}{d t}, \ldots, \frac{d^{R} \vec{q}}{d t^{R}}\right)+\epsilon \frac{d}{d t}(\delta f),
$$

implies

$$
\epsilon \frac{d}{d t}(\delta f)-\sum_{n=0}^{R} \frac{\partial L}{\partial \vec{q}^{(n)}} \delta\left(\frac{d^{n} \vec{q}}{d t^{n}}\right)-\epsilon \dot{g} L=0 .
$$

For $n>0$ the following identity holds

$$
\delta\left(\frac{d^{n} \vec{q}}{d t^{n}}\right)=-\epsilon \sum_{k=0}^{n-1} \frac{d^{k}}{d t^{k}}\left(\dot{g} \vec{q}^{(n-k)}\right)+\epsilon \vec{\chi}^{(n)},
$$


while for $n>1, k>0$ we get

$$
\begin{aligned}
& \frac{d^{k}}{d t^{k}}\left(\dot{g} \vec{q}^{(n-k)}\right) \frac{\partial L}{\partial \vec{q}^{(n)}}=\frac{d}{d t}\left(\sum_{l=0}^{k-1}(-1)^{l} \frac{d^{k-l-1}}{d t^{k-l-1}}\left(\dot{g} \bar{q}^{(n-k)}\right)\left(\frac{d^{l}}{d t^{l}}\right)\left(\frac{\partial L}{\partial \vec{q}^{(n)}}\right)\right)+ \\
&(-1)^{k} \dot{g} \bar{q}^{(n-k)} \frac{d^{k}}{d t^{k}}\left(\frac{\partial L}{\partial \vec{q}^{(n)}}\right) .
\end{aligned}
$$

Using eqs. (32) and (33) one finds

$$
\begin{aligned}
& \sum_{n=1}^{R} \frac{\partial L}{\partial \vec{q}^{(n)}} \delta\left(\frac{d^{n} \vec{q}}{d t^{n}}\right)=-\epsilon \dot{g} \sum_{n=1}^{R} \sum_{k=0}^{n-1}(-1)^{k} \frac{d^{k}}{d t^{k}}\left(\frac{\partial L}{\partial \vec{q}^{(n)}}\right) \vec{q}^{(n-k)}+\epsilon \sum_{n=1}^{R} \frac{\partial L}{\partial \vec{q}^{(n)}} \vec{\chi}^{(n)} \\
& -\epsilon \sum_{n=2}^{R} \sum_{k=1}^{n-1} \frac{d}{d t}\left(\sum_{l=0}^{k-1}(-1)^{l} \frac{d^{k-l-1}}{d t^{k-l-1}}\left(\dot{g} \vec{q}^{(n-k)}\right)\left(\frac{d^{l}}{d t^{l}}\right) \frac{\partial L}{\partial \vec{q}^{(n)}}\right) .
\end{aligned}
$$

On the other hand the Ostrogradski Hamiltonian for $L$ can be written in the form

$$
H=\sum_{l=0}^{R-1} \vec{p}_{l} \vec{q}^{(l+1)}-L,
$$

where

$$
\vec{p}_{n}=\sum_{j=0}^{R-n-1}\left(-\frac{d}{d t}\right)^{j}\left(\frac{\partial L}{\partial \vec{q}^{(n+j+1)}}\right), \quad n=0,1, \ldots, R-1
$$

consequently the first term on the r.h.s. of eq. (34) can be rewritten as follows

$$
\begin{aligned}
& -\epsilon \dot{g} \sum_{n=1}^{R} \sum_{k=0}^{n-1}(-1)^{k} \frac{d^{k}}{d t^{k}}\left(\frac{\partial L}{\partial \vec{q}^{(n)}}\right) \vec{q}^{(n-k)}=-\epsilon \dot{g} \sum_{l=1}^{R} \sum_{j=0}^{R-l}\left(-\frac{d}{d t}\right)^{j}\left(\frac{\partial L}{\partial \vec{q}^{(l+j)}}\right) \vec{q}^{(l)}= \\
& -\epsilon \dot{g} \sum_{l=0}^{R-1} \sum_{j=0}^{R-l-1}\left(-\frac{d}{d t}\right)^{j}\left(\frac{\partial L}{\partial \vec{q}^{(l+j+1)}}\right) \vec{q}^{(l+1)}=-\epsilon \frac{d}{d t}(g H)-\epsilon \dot{g} L,
\end{aligned}
$$


where $H$ is expressed in terms of $\vec{q}$ and their time derivatives. Substituting this result into eq. (34) and using eq. (31) we obtain the following equation

$$
\begin{aligned}
& \epsilon \frac{d}{d t}\left(\delta f+H \dot{g}+\sum_{n=2}^{R} \sum_{k=1}^{n-1} \sum_{l=0}^{k-1}(-1)^{l} \frac{d^{k-l-1}}{d t^{k-l-1}}\left(\dot{g} \vec{q}^{(n-k)}\right)\left(\frac{d^{l}}{d t^{l}}\right) \frac{\partial L}{\partial \vec{q}^{(n)}}\right)- \\
& \epsilon \sum_{n=0}^{R} \frac{\partial L}{\partial \vec{q}^{(n)}} \vec{\chi}^{(n)}=0 .
\end{aligned}
$$

Moreover, one checks that

$$
\sum_{n=0}^{R} \frac{\partial L}{\partial \vec{q}^{(n)}} \vec{\chi}^{(n)}=\frac{d}{d t}\left(\sum_{k=0}^{R-1} \vec{p}_{k} \vec{\chi}^{(k)}\right)+\vec{\chi} \sum_{k=0}^{R}\left(-\frac{d}{d t}\right)^{k}\left(\frac{\partial L}{\partial \vec{q}^{(k)}}\right) .
$$

Together with eq. (38) this leads to the following integral of motion

$$
C=H g-\sum_{k=0}^{R-1} \vec{p}_{k} \vec{\chi}^{(k)}+\sum_{n=2}^{R} \sum_{k=1}^{n-1} \sum_{l=0}^{k-1} \frac{d^{k-l-1}}{d t^{k-l-1}}\left(\dot{g} \vec{q}^{(n-k)}\right)\left(-\frac{d^{l}}{d t^{l}}\right)\left(\frac{\partial L}{\partial \vec{q}^{(n)}}\right)+\delta f .
$$

Now, let us apply these general formulae to our Lagrangian (11) and symmetry transformations (24)-(27). In this case the generalized momenta (36) and the Hamiltonian $H$, when written in terms of $\vec{q}$ s, read

$$
\begin{gathered}
\vec{p}_{n}=m(-1)^{\frac{N-1}{2}-n} \vec{q}^{(N-n)}, \quad n=0,1, \ldots, \frac{N-1}{2}, \\
H=\sum_{n=0}^{\frac{N-3}{2}}(-1)^{\frac{N-1}{2}-n} \vec{q}^{(N-n)} \vec{q}^{(n+1)}+\frac{m}{2}\left(\vec{q}^{\left(\frac{N+1}{2}\right)}\right)^{2} .
\end{gathered}
$$

Let us now find the integrals of motion. For the transformations (24), $g_{k}=0$ and $\vec{\chi}_{k}=(-1)^{k-\frac{N+1}{2}} t^{k}, k=0, \ldots, N$ while the functions $\delta \vec{f}_{k}$ are of the form (see Appendix, eq. (56) for small $\vec{x}$ )

$$
\begin{aligned}
& \delta \vec{f}_{k}=0, k=0, \ldots, \frac{N-1}{2} ; \\
& \delta \vec{f}_{k}=m \sum_{n=\frac{N+1}{2}}^{k}(-t)^{k-n} \frac{k !}{(k-n) !} \vec{q}^{(N-n)}, k=\frac{N+1}{2}, \ldots, N .
\end{aligned}
$$


Due to eq. (40) the corresponding integrals of motion are of the form

$$
\vec{C}_{k}=m \sum_{n=0}^{k}(-t)^{k-n} \frac{k !}{(k-n) !} \vec{q}^{(N-k)}, k=0, \ldots, N .
$$

For time translations obviously $C=H$.

Similarly, for dilatations (26),$\delta f=0$. Substituting $g=-t, \vec{\chi}=\frac{-N}{2} \vec{q}$ into eq. (40) we obtain the following integral of motion

$$
D=-t H+D(t)=-t H+m \sum_{k=0}^{\frac{N-1}{2}}(-1)^{\frac{N-1}{2}-k}\left(\frac{N}{2}-k\right) \vec{q}^{(N-k)} \vec{q}^{(k)} .
$$

For conformal transformations (27), eq. (58) implies $\delta f=\frac{m}{2}\left(\frac{N+1}{2}\right)^{2}\left(\vec{q}^{\left(\frac{N-1}{2}\right)}\right)^{2}$. Moreover $g=t^{2}$ and $\vec{\chi}=t N \vec{q}$. Using this we find, after some computations, the corresponding integral of motion

$$
\begin{aligned}
& K=t^{2} H-2 t D(t)+K(t)=t^{2} H-2 t D(t)+ \\
& \quad m \sum_{j=0}^{\frac{N-3}{2}}(j+1)(N-j)(-1)^{\frac{N-1}{2}-j} \bar{q}^{(j)} \vec{q}^{(N-j+1)}+\frac{m}{2}\left(\frac{N+1}{2}\right)^{2}\left(\vec{q}^{\left(\frac{N-1}{2}\right)}\right)^{2} .
\end{aligned}
$$

Finally, by considering rotations one gets the following expression for angular momentum

$$
\vec{J}=m \sum_{k=0}^{\frac{N-1}{2}}(-1)^{\frac{N-1}{2}-k} \vec{q}^{(k)} \times \vec{q}^{(N-k)} .
$$

Concluding, let us note that, as in the case of first order theory, all integrals of motion (44)-(47) can be obtained from the ones on the Hamiltonian level (see, eqs. (6)) by expressing Ostrogradski momenta (41) in terms of $\vec{q}$ and it time derivatives.

\section{Two-dimensional case}

As we have mentioned, in the case of dimension 2 and $N$ even, there also exists the central extension of $N$-GCA (11) and (2). It is given by

$$
\left[C_{j}^{a}, C_{k}^{b}\right]=-i \epsilon^{a b} \delta^{N, j+k}(-1)^{\frac{j-k}{2}} k ! j ! M,
$$


where $\quad a, b=1,2, \quad j, k=0,1, \ldots, N$. Neglecting "internal" degrees of freedom general Hamiltonian systems with this symmetry is of the form (see, [1])

$$
\begin{aligned}
\left\{q_{j}^{a}, p_{k}^{b}\right\} & =\delta^{a b} \delta_{j k}, \quad j, k=0, \ldots, \frac{N}{2}-1, \quad a, b=1,2 ; \\
\left\{q_{\frac{N}{2}}^{a}, q_{\frac{N}{2}}^{b}\right\} & =\frac{1}{m} \epsilon^{b a}, \quad a, b=1,2 .
\end{aligned}
$$

Denoting $p_{\frac{N}{2}}^{a}=\frac{m}{2} \epsilon^{b a} q_{\frac{N}{2}}^{b}$ and

$$
\begin{aligned}
h(t) & =\sum_{k=0}^{\frac{N}{2}-1} \vec{p}_{k} \vec{q}_{k+1}, \\
d(t) & =\sum_{k=0}^{\frac{N}{2}-1}\left(\frac{N}{2}-k\right) \vec{p}_{k} \vec{q}_{k}, \\
k(t) & =-\sum_{k=1}^{\frac{N}{2}-1}(N-k+1) k \vec{p}_{k} \vec{q}_{k-1}-N\left(\frac{N}{2}+1\right) \vec{q}_{\frac{N}{2}-1} \vec{p}_{\frac{N}{2}}, \\
j(t) & =\sum_{k=0}^{\frac{N}{2}} \vec{q}_{k} \times \vec{p}_{k},
\end{aligned}
$$

one can find Noether's charges:

$$
\begin{aligned}
h & =h(t), \\
d & =d(t)-t h(t), \\
k & =k(t)-2 t d(t)+t^{2} h(t), \\
j & =j(t), \\
c_{j}^{a} & =\left\{\begin{array}{c}
(-1)^{j+\frac{N}{2}+1} \sum_{k=0}^{j} \frac{j !}{(j-k) !} t^{j-k} p_{k}^{a}, \quad 0 \leq j \leq \frac{N}{2}-1 \\
(-1)^{j+\frac{N}{2}+1} \sum_{k=0}^{\frac{N}{2}-1} \frac{j !}{(j-k) !} t^{j-k} p_{k}^{a}+ \\
m \sum_{k=\frac{N}{2}}^{j}(-1)^{j-k} \frac{j !}{(j-k) !} t^{j-k} \epsilon_{a b} q_{N-k}^{b}, \quad \frac{N}{2} \leq j \leq N .
\end{array}\right.
\end{aligned}
$$

Of course, we can repeat the preceding considerations. For example, infinitesimal transformations of $\vec{q}_{0}$ are of the form: 
- for $\vec{c}_{j}$ :

$$
\delta \vec{q}_{0}=\left\{\sum_{j} \vec{x}_{j} \vec{c}_{j}, \vec{q}_{0}\right\}=\sum_{j=0}^{N} t^{j}(-1)^{j-\frac{N}{2}} \vec{x}_{j},
$$

- for $h$ :

$$
\delta \vec{q}_{0}=\left\{\tau h, \vec{q}_{0}\right\}=-\dot{\vec{q}}_{1}
$$

- for $d$ :

$$
\delta \vec{q}_{0}=\left\{\lambda d, \vec{q}_{0}\right\}=\lambda\left(t \vec{q}_{1}-\frac{N}{2} \vec{q}_{0}\right)
$$

- for $k$ :

$$
\delta \vec{q}_{0}=\left\{c k, \vec{q}_{0}\right\}=c\left(t \vec{q}_{0} N-t^{2} \vec{q}_{1}\right) .
$$

In terms of the variable $\vec{q}=\vec{q}_{0}$ the Lagrangian corresponding to the Ostrogradski Hamiltonian $h$ reads

$$
L=\frac{m}{2} \epsilon_{a b} \frac{d^{\frac{N-1}{2}} q^{a}}{d t^{\frac{N-1}{2}}} \frac{d^{\frac{N+1}{2}} q^{b}}{d t^{\frac{N+1}{2}}} .
$$

As previously, one can show that the action of $N$-GCA can be translated into coordinate space $\vec{q}$ and the symmetry transformations, corresponding to (52), are of the form (cf. eqs. (24)-(27) for the case of $N$ odd)

$$
\begin{gathered}
t^{\prime}=t, \quad \overrightarrow{q^{\prime}}\left(t^{\prime}\right)=\vec{q}(t)+\sum_{k=0}^{N}(-1)^{k-\frac{N}{2}} t^{k} \vec{x}_{k} ; \\
t^{\prime}=t+\tau, \quad \overrightarrow{q^{\prime}}\left(t^{\prime}\right)=\vec{q}(t) ; \\
t^{\prime}=e^{-\lambda} t, \quad \overrightarrow{q^{\prime}}\left(t^{\prime}\right)=e^{-\lambda \frac{N}{2}} \vec{q}(t) ; \\
t^{\prime}=\frac{t}{1-c t}, \quad \overrightarrow{q^{\prime}}\left(t^{\prime}\right)=\frac{\vec{q}(t)}{(1-c t)^{N}}
\end{gathered}
$$

Differential realization of the algebra is the same as in the case of $N$ odd (there is not central extension on Lagrangian level). 


\section{Conclusions}

In this paper we completed the picture by providing dynamical realization of $N$-Galilean conformal symmetries, in the case when the relevant algebra admits central extension. It has been shown previously [1, 6], that the generic Hamiltonian system on which the $N$-Galilean conformal group acts transitively as a group of canonical transformation is described by Ostrogradski Hamiltonian (modulo the dynamics of "internal" degrees of freedom). Here we defined the point transformations acting on coordinate space which coincide "on-shell" with the above canonical ones. They provide the Noether

symmetries of free Lagrangian containing $\frac{N+1}{2}$-th order time derivatives as well as the Noether's charges coinciding with those obtained on Hamiltonian level. It can be further shown [11] that $N$-Galilean conformal group is the maximal group of Noether symmetries of free higher-derivative Lagrangian. This generalizes the Niederer's result [9] concerning the Schrödinger group, $N=1$ (actually, Niederer proved it on quantum level)

Acknowledgments The authors would like to thank Cezary Gonera, Piotr Kosiński and Paweł Maślanka for helpful discussions and useful remarks. This work is supported in part by MNiSzW grant No. N202331139.

\section{Appendix}

Let us now show explicitly that under the transformations of $N$-Galilean conformal group the Lagrangian (11) transforms by total derivative; more precisely we prove that

$$
\frac{m}{2}\left(\frac{d^{\frac{N+1}{2}} \overrightarrow{q^{\prime}}}{d t^{\prime \frac{N+1}{2}}}\right)^{2} \frac{d t^{\prime}}{d t}=\frac{m}{2}\left(\frac{d^{\frac{N+1}{2}} \vec{q}}{d t^{\frac{N+1}{2}}}\right)^{2}+\frac{d f}{d t}
$$

where $f$ is a function of $\vec{q}$ and its time derivatives up to $\frac{N-1}{2}$ order. Using the formulae of Sec. 2 we see that $f=0$ for time translations, dilatations 
and rotations. For transformations (24) the functions $f_{k}$ are of the form

$$
\begin{aligned}
& f_{k}=0 \quad k=0, \ldots, \frac{N-1}{2} \\
& f_{k}=m \sum_{n=\frac{N+1}{2}}^{k}(-t)^{k-n} \frac{k !}{(k-n) !} \vec{q}^{(N-n)} \vec{x}+ \\
& \frac{m}{2}\left(\frac{k !}{\left(k-\frac{N+1}{2}\right) !}\right)^{2} \frac{1}{2 k-N} t^{2 k-N} \vec{x}^{2}, \quad k=\frac{N+1}{2}, \ldots, N .
\end{aligned}
$$

For the conformal transformation (27) the condition (155) takes the form

$$
\begin{aligned}
& \frac{m}{2}\left(\frac{d^{\frac{N+1}{2}} \overrightarrow{q^{\prime}}}{d t^{\prime \frac{N+1}{2}}}\right)^{2} \frac{d t^{\prime}}{d t}-\frac{m}{2}\left(\frac{d^{\frac{N+1}{2}} \vec{q}}{d t^{\frac{N+1}{2}}}\right)^{2}= \\
& \sum_{\substack{l, l^{\prime}=0 \\
l+l^{\prime}<N+1}}^{\frac{N+1}{2}} \frac{\left(\frac{N+1}{2}\right)^{2}(N-l) !\left(N-l^{\prime}\right) !\left(c^{N+1-l-l^{\prime}}\right.}{l !\left(\frac{N+1}{2}-l\right)\left(\frac{N+1}{2}-l^{\prime}\right) !(1-c t)^{N+1-l-l^{\prime}}} \vec{q}^{(l)} \vec{q}^{\left(l^{\prime}\right)} .
\end{aligned}
$$

Let us put

$$
f=\frac{m}{2}\left(\frac{N+1}{2}\right)^{2} \sum_{l, l^{\prime}=0}^{\frac{N-1}{2}} \frac{a\left(l, l^{\prime}\right) c^{N-l-l^{\prime}}}{(1-c t)^{N-l-l^{\prime}}} \vec{q}^{(l)} \bar{q}^{\left(l^{\prime}\right)} .
$$

Then eqs. (57) and (58) imply

$$
\left(N-l-l^{\prime}\right) a\left(l, l^{\prime}\right)+a\left(l-1, l^{\prime}\right)+a\left(l, l^{\prime}-1\right)=\frac{(N-l) !\left(N-l^{\prime}\right) !}{l ! l^{\prime} !\left(\frac{N+1}{2}-l\right)\left(\frac{N+1}{2}-l^{\prime}\right) !} .
$$

Eq. (55) is established once we show that this recurrence has the solution. To this end we put

$$
a\left(l, l^{\prime}\right)=\left(N-l-l^{\prime}-1\right) ! d\left(l, l^{\prime}\right) .
$$

In terms of $d\left(l, l^{\prime}\right)$ eq. (59) reads

$$
d\left(l, l^{\prime}\right)+d\left(l-1, l^{\prime}\right)+d\left(l, l^{\prime}-1\right)=\frac{1}{N !} C_{l l^{\prime}}^{N} \prod_{k=\frac{N+3}{2}}^{N}(k-l) \prod_{k^{\prime}=\frac{N+3}{2}}^{N}\left(k^{\prime}-l^{\prime}\right),
$$


where

$$
C_{l_{1} l_{2}}^{n}=\frac{n !}{l_{1} ! l_{2} !\left(n-l_{1}-l_{2}\right) !} .
$$

Note that the following identity holds

$$
C_{l_{1} l_{2}}^{n}+C_{\left(l_{1}-1\right) l_{2}}^{n}+C_{l_{1}\left(l_{2}-1\right)}^{n}=C_{l_{1} l_{2}}^{n+1} .
$$

The product $\prod_{k=\frac{N+3}{2}}^{N}(k-l)$ is a polynomial in $l$ of degree $\frac{N-1}{2}$ with $N$ dependent coefficients. It is easy to show that it can be rewritten as

$$
\prod_{k=\frac{N+3}{2}}^{N}(k-l)=\sum_{n=0}^{\frac{N+1}{2}} \beta_{n}(N) \prod_{k=0}^{n-1}(l-k)
$$

for some $\beta_{n}(N)$ 's. Using (64) one can rewrite eq. (61) as

$$
d\left(l, l^{\prime}\right)+d\left(l-1, l^{\prime}\right)+d\left(l, l^{\prime}-1\right)=\sum_{n, n^{\prime}=0}^{\frac{N-1}{2}} \gamma_{n, n^{\prime}}(N) C_{l-n}^{N-n-n^{\prime}-n^{\prime}}
$$

By virtue of the identity (63) we find

$$
d\left(l, l^{\prime}\right)=\sum_{n, n^{\prime}=0}^{\frac{N-1}{2}} \gamma_{n, n^{\prime}}(N) C_{l-n l^{\prime}-n^{\prime}}^{N-n-n^{\prime}-1}
$$

so eq.(55) holds.

\section{References}

[1] K. Andrzejewski, J. Gonera, P. Maślanka, Phys. Rev. D86 (2012), 065009

[2] A. Kirillov, Elements of the Theory of Representations, Springer 1976 ;

J.M. Soriau, Structure of Dynamical Systems. A Symplectic View of Physics, Birkhauser 1997;

V.I. Arnold, Mathematical Methods of Classical Mechanics, Springer 1989;

R. Giachetti, Riv. Nuovo. Cim. 4, (1981), 1; 
[3] P. Havas, J. Plebański, J. Math. Phys. 19 (1978), 482;

M. Henkel, J. Stat. Phys. 75 (1994), 1023;

J. Negro, M.A. del Olmo, A. Rodriguez-Marco, J. Math. Phys. 38 (1997), 3786, 3810;

[4] D. Martelli, Y. Tachikawa, JHeP 05, (2010), 091;

[5] A.V. Galajinsky, I. Masterov, Phys. Lett. B702 (2011), 265;

[6] J. Gomis, K. Kamimura, Phys. Rev. D85 (2012), 045023;

[7] J. Lukierski, P.C. Stichel, W.J. Zakrzewski, Phys. Lett. A357 (2006), 3810;

[8] C. Duval, P.A. Horvathy, J. Phys. A42 (2009), 465206;

[9] U.Niederer, Helv. Phys. Acta. 45 (1973), 802;

[10] M. Ostrogradski, Mem. Acad. St. Petersburg 4 (1850), 385;

J. Govaerts, M.S. Rashid, preprint, arXiv hep-th/9403009;

K. Andrzejewsiki, J. Gonera, P. Machalski, P. Maślanka, Phys. Rev. D82 (2010), 045008;

[11] K. Andrzejewski, J. Gonera, preprint, arXiv:1209.5884; 